\title{
The restrictive-obstructive continuum and the failing heart
}

\section{Gunnar Engström}

The close interrelationship between the heart and lungs was early recognised by medical scientists. In a paper from 1895, Alfred Lee Loomis stated that dyspnoea could be divided into four categories on an aetiological basis: laryngeal dyspnoea, bronchial dyspnoea, pulmonic dyspnoea and cardiac dyspnoea. The term cardiac dyspnoea was used if dyspnoea arose 'from arrest in the passage of blood through the heart to the lungs'. ${ }^{1}$ Early studies using spirometry observed that patients with heart disease had lower vital capacity than 'normal individuals'. ${ }^{2}$ Many years later, prospective analyses of population-based cohorts showed that reduced lung function also could predict the onset of cardiac diseases. ${ }^{3}{ }^{4}$ Many studies since have shown that reduced vital capacity or $\mathrm{FEV}_{1}$ is associated with increased incidence of cardiovascular diseases among individuals from the general population. ${ }^{56}$ The relationships have been quite strong and comparable with the effects of traditional cardiovascular risk factors, such as obesity or hyperlipidaemia. The increased risk is not limited to those with the smallest lung volumes; in many studies there is a dose-response relationship over the whole distribution of $\mathrm{FEV}_{1}$ or FVC.

However, even though much research has been performed over the past decades, the underlying causal reasons for the increased cardiovascular risk remain elusive. Cardiovascular and pulmonary diseases certainly share some important risk factors, such as smoking, inflammation and physical inactivity, but the increased risk in individuals with low lung function has usually persisted after extensive adjustments for other risk factors, and significant relationships have been shown in studies of never-smokers. ${ }^{6}$ It has been reported that cardiovascular disease and reduced lung function share common genetic determinants, but this probably cannot explain more than a small fraction of the excess risk. ${ }^{7}$

The fact that cardiovascular disease is a very heterogeneous concept further increases the complexity of the interactions

Correspondence to Professor Gunnar Engström, Department of Clinical Science, Lund University, Jan Waldenströms gata 35, CRC 60:13, Malmö 20502, Sweden; Gunnar.Engstrom@med.lu.se with lung function. Reduced $\mathrm{FEV}_{1}$ or FVC has been linked to quite different cardiovascular complications, such as myocardial infarction and stroke, ${ }^{5}{ }^{6}$ ventricular arrhythmia, ${ }^{8}$ incidence of atrial fibrillation, ${ }^{9}$ diabetes mellitus ${ }^{10}$ and blood pressure variability. ${ }^{11}$

Similarly, 'reduced lung function' includes many different phenotypes and more research is needed to clarify which aspects of lung function impairment are most important. Even though there are several exceptions in the literature, it is noteworthy that many population-based studies have reported increased risk for individuals with low $\mathrm{FEV}_{1}$ or FVC, well within the normal range, but no clear relationship with low $\mathrm{FEV}_{1} / \mathrm{FVC}^{10}{ }^{12}$ Since COPD is characterised by low $\mathrm{FEV}_{1} /$ FVC ratio, this is in contrast with the findings in many clinical settings, which often have reported high prevalence of cardiovascular disease co-morbidity in patients with COPD. ${ }^{13}$ Indeed, there is reason to believe that the risk of cardiovascular diseases is associated with more than one type of lung function impairment. A recent study reported that the natural history of airway obstruction leading to COPD could be quite different; some individuals had a rapid $\mathrm{FEV}_{1}$ decline while others had low $\mathrm{FEV}_{1}$ already in early adulthood. ${ }^{14}$ The natural history leading to cardiovascular disease could similarly involve different pathways and much more research is needed to fully characterise the phenotypes with high risk. Prospective studies of cohorts with detailed information about respiratory function have the potential to add new and important insights into the natural history of both cardiovascular and respiratory diseases.

The comorbidity between COPD and heart failure has received much attention in the past few years. However, few studies have explored whether reduced lung function predicts future onset of heart failure. In the current issue of Thorax, Wannamethee et $a l^{15}$ assessed the relationships between lung function, circulating cardiac markers and incidence of heart failure, in 3242 older men. The research was performed in the British Regional Heart study, a well-known population-based cohort, which was established with the purpose of studying cardiovascular disease and its risk factors. The authors show that N-terminal probrain natriuretic peptide (NT-proBNP) and troponin $\mathrm{T}$, markers of myocardial wall stress and myocardial injury, are inversely associated with $\mathrm{FEV}_{1}$ and FVC. Both cardiac markers were increased in men with restrictive pattern on spirometry as well as for those with moderate or severe obstruction. Incidence of heart failure was increased in men with moderate or severe obstruction, while the increased incidence in men with pulmonary restriction was attenuated and nonsignificant after adjustments for risk factors. The same pattern was seen when the analyses were restricted to never smokers and long-term ex-smokers. NT-proBNP and troponin $\mathrm{T}$ are sensitive measures of preclinical cardiac injury and adjustments for these markers should substantially reduce the risk of reverse confounding in this study, that is, that preclinical heart failure caused the abnormal findings on spirometry. The increased levels of cardiac markers both in the restrictive and obstructive ends of the continuum illustrate that the cardiovascular risk could have at least two distributions. Since low FVC is associated with increased risk of diabetes, ${ }^{10}$ it could be speculated that the restrictive pattern represents a 'metabolic' risk group, which also may be linked to early life exposures, ${ }^{16}$ while the obstructive pattern could be acquired later in life and represent other pathways to cardiovascular disease.

The men were 60-79 years old at baseline and were followed up over a mean time of 13 years. One question, acknowledged by the authors, is whether the results can be generalised to younger age groups. The fixed ratio between $\mathrm{FEV}_{1}$ and FVC was used both for the definition of obstruction according to Global Initiative for Chronic Obstructive Lung Disease $\left(\mathrm{FEV}_{1} / \mathrm{FVC}<0.70\right)$ and to define restrictive airway pattern $\left(\mathrm{FEV}_{1} / \mathrm{FVC} \geq 0.70\right.$ and FVC $\%$ predicted $<80 \%)$. Since $\mathrm{FEV}_{1} /$ FVC declines with increasing age, it is likely that the proportions of and characteristics of men fulfilling the obstructive and restrictive definitions depend on the age group of the study. Hence, further studies of women and younger populations are of interest.

The causal relationships between lung function and incidence of cardiovascular disease are complex and elusive and much research is still needed to clarify the nature of the associations. The study by Wannamethee et al has taken us one step further by their report of cardiac markers 


\section{Editorial}

and incidence of heart failure along the restrictive-obstructive continuum of pulmonary function.

Competing interests None declared.

Provenance and peer review Commissioned; externally peer reviewed.

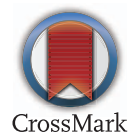

To cite Engström G. Thorax 2016;71:487-488.

Published Online First 16 February 2016

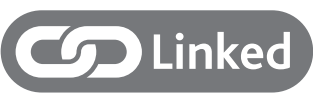

http://dx.doi.org/10.1136/thoraxjnl-2014-206724

Thorax 2016;71:487-488.

doi:10.1136/thoraxjnl-2015-208119

\section{REFERENCES}

1 Lee Loomis A. Cardiac Dyspnea. Trans Am Climatol Assoc 1895;10:114-20.
2 Calhoun JA, Cullen GE, Harrison TR, et al. Studies in congestive heart failure: XIV. Orthopnea: its relation to ventilation, vital capacity, oxygen saturation and acid-base condition of arterial and jugular blood. J Clin Invest 1931;10:833-55.

3 Friedman GD, Klatsky AL, Siegelaub AB. Lung function and risk of myocardial infarction and sudden cardiac death. N Engl J Med 1976;294:1071-5.

4 Kannel WB, Seidman JM, Fercho W, et al. Vital capacity and congestive heart failure. The Framingham study. Circulation 1974;49:1160-6.

5 Young RP, Hopkins R, Eaton TE. Forced expiratory volume in one second: not just a lung function test but a marker of premature death from all causes. Eur Respir J 2007;30:616-22.

6 Hole DJ, Watt GC, Davey-Smith G, et al. Impaired lung function and mortality risk in men and women: findings from the Renfrew and Paisley prospective population study. BMJ 1996;313:711-15; discussion 715-6.

7 Sabater-Lleal M, Mälarstig A, Folkersen L, et al. Common genetic determinants of lung function, subclinical atherosclerosis and risk of coronary artery disease. PLOS ONE 2014;9:e104082.

8 Engström G, Wollmer $P$, Hedblad B, et al. Occurrence and prognostic significance of ventricular arrhythmia is related to pulmonary function: a study from "men born in 1914," Malmö, Sweden. Circulation 2001;103:3086-91.

9 Johnson LS, Juhlin T, Engström G, et al. Reduced forced expiratory volume is associated with increased incidence of atrial fibrillation: the Malmo Preventive Project. Europace 2014;16:182-8.

10 Klein OL, Krishnan JA, Glick S, et al. Systematic review of the association between lung function and Type 2 diabetes mellitus. Diabet Med 2010;27:977-87.

11 Engström G, Gerhardsson de Verdier M, Dahlbäck M, et al. Blood pressure variability and cardiovascular autonomic function in relation to forced expiratory volume: a population-based study. Chest 2009;136:177-83.

12 Schroeder EB, Welch VL, Couper D, et al. Lung function and incident coronary heart disease: the Atherosclerosis Risk in Communities Study. Am J Epidemiol 2003;158:1171-81.

13 Müllerova H, Agusti A, Erqou S, et al. Cardiovascular comorbidity in COPD: systematic literature review. Chest 2013;144:1163-78.

14 Lange $\mathrm{P}$, Celli B, Agustí $\mathrm{A}$, et al. Lung-function trajectories leading to chronic obstructive pulmonary disease. N Engl J Med 2015;373:111-22.

15 Wannamethee SG, Shaper AG, Papacosta O, et al. Lung function and airway obstruction: associations with circulating markers of cardiac function and incident heart failure in older men-the British Regional Heart Study. Thorax 2016;71:526-34.

16 Baumann S, Godtfredsen NS, Lange P, et al. The impact of birth weight on the level of lung function and lung function decline in the general adult population. The Inter99 study. Respir Med 2015;109:1293-9. 